Cahiers $d u$ MONDE RUSSE

\section{Cahiers du monde russe}

Russie - Empire russe - Union soviétique et États indépendants

$48 / 4 \mid 2007$

Varia

\title{
Oxana Stuppo, Das Feindbild als zentrales Element der Kommunikation im Spätstalinismus
}

\author{
Наталья В. Суржикова - Natal'ja V. Suržikova
}

\section{OpenEdition}

Journals

Édition électronique

URL : https://journals.openedition.org/monderusse/6095

DOI : 10.4000/monderusse.6095

ISSN : $1777-5388$

Éditeur

Éditions de l'EHESS

\section{Édition imprimée}

Date de publication : 2 décembre 2007

Pagination : 755-761

ISBN : 978-2-7132-2148-4

ISSN : $1252-6576$

Référence électronique

Наталья В. Суржикова - Natal'ja V. Suržikova, «Oxana Stuppo, Das Feindbild als zentrales Element der Kommunikation im Spätstalinismus», Cahiers du monde russe [Онлайн], 48/4 | 2007, Выложить онлайн 28 décembre 2009, Наводить справки в 03 septembre 2022. URL: http://

journals.openedition.org/monderusse/6095 ; DOl: https://doi.org/10.4000/monderusse.6095

Ce document a été généré automatiquement le 3 septembre 2022.

All rights reserved 


\title{
Oxana Stuppo, Das Feindbild als zentrales Element der Kommunikation im Spätstalinismus
}

\author{
Наталья В. Суржикова - Natal'ja V. Suržikova
}

\section{RÉFÉRENCE}

Oxana STUPро, Das Feindbild als zentrales Element der Kommunikation im

Spätstalinismus. Der Fall Sverdlovsk 1945-1953. Wiesbaden : Harrassowitz Verlag, 2007, 224 p. (Forschungen zur osteuropäischen Geschichte, 70)

1 ОБРАЩЕНИЕ СОЦИОЛОГОВ, ПСИХОЛОГОВ И ИСТОРИКОВ К ПОНЯТИЮ «ОБРАЗ ВРАГА», ОЧЕВИДНО, СЛЕДУЕТ РАССМАТРИВАТЬ КАК СЛЕДСТВИЕ АНТРОПОЛОГИЗАЦИИ НАУКИ, КОТОРАЯ, НАРЯДУ С ИЗУЧЕНИЕМ ФАКТА ИСТОРИИ В ЕГО ТРАДИЦИОННОМ ПОНИМАНИИ, ПРЕДПОЛАГАЕТ ИССЛЕДОВАНИЕ МИРА ЧЕЛОВЕКА, ЕГО ОРИЕНТАЦИЙ И МОТИВОВ, ПОЛУЧАЮЩИХ СВОЕ ВОПЛОЩЕНИЕ В СПЕЦИФИЧЕСКИХ МЫСЛИТЕЛЬНЫХ, А ЗАТЕМ И ПОВЕДЕНЧЕСКИХ МАТРИЦАХ, КОНТУРЫ КОТОРЫХ МЕНЯЮТСЯ СО СМЕНОЙ ИСТОРИЧЕСКИХ ВЕХ.

КОНКРЕТИЗАЦИИ И КОНЦЕПТУАЛИЗАЦИИ ИССЛЕДОВАНИЙ, ПОСВЯЩЕННЫХ ТАКОМУ СОЦИАЛЬНО-ПСИХОЛОГИЧЕСКОМУ КОНСТРУКТУ, КАК «ОБРАЗ ВРАГА», БУДЕТ СПОСОБСТВОВАТЬ РАБОТА О. СТУППО, ЗАКРЫВАЮЩАЯ ЕСЛИ НЕ ОДНУ ИЗ ПРОБЛЕМНЫХ, ТО, БЕССПОРНО, ОДНУ ИЗ ГЕОГРАФИЧЕСКИХ ЛАКУН РЕТРОСПЕКТИВНОЙ ТЕМАТИКИ. ПРЕДМЕТОМ ИССЛЕДОВАТЕЛЬСКОГО ИНТЕРЕСА В ДАННОМ КОНКРЕТНОМ СЛУЧАЕ СТАЛО ФОРМИРОВАНИЕ ОБРАЗА ВРАГА В СОВЕТСКОМ ОБЩЕСТВЕ ПОЗДНЕСТАЛИНСКОГО ОБРАЗЦА И ЕГО ЦИРКУЛЯЦИЯ В ПРЕДЕЛАХ РЕГИОНА, ОГРАНИЧЕННОГО В АДМИНИСТРАТИВНО-ТЕРРИТОРИАЛЬНОМ ПЛАНЕ РАМКАМИ СВЕРДЛОВСКОЙ ОБЛАСТИ, ЧТО В СМЫСЛЕ ГЕОГРАФИЧЕСКОМ ТОЖДЕСТВЕННО ПРЕДЕЛАМ СРЕДНЕГО УРАЛА. ТРУДНО НЕ СОГЛАСИТЬСЯ С АВТОРОМ В ТОМ, ЧТО ПОЗДНЕСТАЛИНСКИЙ ПЕРИОД ИСТОРИИ СССР НЕ ТАК АКТИВНО ИССЛЕДУЕТСЯ, КАК ОН ТОГО ЗАСЛУЖИВАЕТ, БУДУЧИ 
ТЕМ САМЫМ ВРЕМЕННЫМ ОТРЕЗКОМ, КОГДА СУЩНОСТНЫЕ ХАРАКТЕРИСТИКИ СТАЛИНИЗМА КАК ИДЕОЛОГИИ, РЕЖИМА И ЭТАПА ОБЩЕСТВЕННОГО РАЗВИТИЯ ПРОЯВИЛИСЬ НАИБОЛЕЕ ОТЧЕТЛИВО. 1945-1953 ГОДЫ, НЕ РЕДКО НОМИНИРУЕМЫЕ В ЛИТЕРАТУРЕ КАК АПОГЕЙ СТАЛИНИЗМА, СТАЛИ ЛОКУСОМ ЗРЕЛЫХ ЦЕННОСТЕЙ И АНТИЦЕННОСТЕЙ ЭПОХИ, ВКЛЮЧАЯ ЦЕННОСТНЫЕ КЛАСТЕРЫ КОММУНИКАТОРНОГО СВОЙСТВА.

КОНЦЕПТООБРАЗУЮЩИМИ ДЛЯ ИССЛЕДОВАНИЯ О.СТУППО ЯВЛЯЮТСЯ СЛЕДУЮЩИЕ ДОПУЩЕНИЯ. ВО-ПЕРВЫХ, ОБРАЗ ВРАГА РАССМАТРИВАЕТСЯ АВТОРОМ КАК ЩПРОДУКТ СТАЛИНСКОЙ СИСТЕМЫ», СОЗДАВАЕМЫЙ В СОАВТОРСТВЕ С НАСЕЛЕНИЕМ, ЧТО ПРЕВРАЩАЛО ЕГО (ОБРАЗ ВРАГА) В ЦЕНТРАЛЬНЫЙ ЭЛЕМЕНТ В СТРУКТУРЕ КОММУНИКАЦИЙ, ПРИЗВАННЫЙ ИГРАТЬ УНИФИЦИРУЮЩУЮ, ГОМОГЕНИЗИРУЮЩУЮ РОЛЬ, - РОЛЬ «ДЕЗАКТИВАТОРА», «НЕЙТРАЛИЗАТОРА» ПОТЕНЦИАЛЬНО ВОЗМОЖНОЙ ИНВАРИАНТНОСТИ МНЕНИЙ ИЛИ ОБРАЗОВ ЖИЗНИ. ВТОРЫМ ВАЖНЕЙШИМ ПОСЫЛОМ ЯВЛЯЕТСЯ ИССЛЕДОВАТЕЛЬСКАЯ ГИПОТЕЗА О ТОМ, ЧТО ОБРАЗ ВРАГА НЕ СОСТАВЛЯЛ ОРГАНИЧЕСКОЙ ЦЕЛОСТНОСТИ, И В СОЦИАЛЬНО-ПОЛИТИЧЕСКИХ ПРАКТИКАХ ИМЕЛИ ХОЖДЕНИЕ» РАЗНЫЕ ОБРАЗЫ ВРАГА, ПАРАЛЛЕЛЬНОЕ ФУНКЦИОНИРОВАНИЕ КОТОРЫХ, В ЧАСТНОСТИ, ОПРЕДЕЛЯЛОСЬ НЕРЕЛЕВАНТНОСТЬЮ УСЛОВИЙ КОММУНИЦИРОВАНИЯ В СОВЕТСКОМ ГОРОДЕ И В СОВЕТСКОЙ ДЕРЕВНЕ, ЧТО, В СВОЮ ОЧЕРЕДЬ, БЫЛО ОБУСЛОВЛЕНО РАЗНЫМ КУЛЬТУРНЫМ УРОВНЕМ И РАЗНЫМИ ПОТРЕБНОСТЯМИ РАБОЧЕГО И КОЛХОЗНИКА. В-ТРЕТЬИХ, ЗА НЕВОЗМОЖНОСТЬЮ ДИФФЕРЕНЦИАЦИИ ОБРАЗОВ ВНЕШНЕПОЛИТИЧЕСКИХ И ВНУТРИПОЛИТИЧЕСКИХ ВРАГОВ АВТОР СРАЗУ ОТКАЗЫВАЕТСЯ ОТ ИХ АВТОНОМНОГО РАССМОТРЕНИЯ, В ОСНОВНОМ, МЕЖДУ ТЕМ, ФОКУСИРУЯСЬ НА ВРАГАХ, А ТОЧНЕЕ НА ОБРАЗАХ ВНУТРЕННИХ ВРАГОВ. В-ЧЕТВЕРТЫХ, ГЛУБИНЕ ИССЛЕДОВАНИЯ ТЕОРЕТИЧЕСКИХ И КОНКРЕТНО-ИСТОРИЧЕСКИХ АСПЕКТОВ ТЕМЫ, А ИМЕННО НАЗНАЧЕНИЯ И ЛОГИКИ, МЕХАНИЗМОВ ФОРМИРОВАНИЯ И ФУНКЦИЙ ОБРАЗА ВРАГА, ЧТО, СОБСТВЕННО, И ЯВЛЯЕТСЯ ЦЕЛЬЮ РАБОТЫ, ПО МНЕНИЮ АВТОРА, ДОЛЖНО СПОСОБСТВОВАТЬ ЕГО РАССМОТРЕНИЕ КАК КОМПЛЕКСНОГО, «МЕЖОТРАСЛЕВОГО» ОБРАЗОВАНИЯ, ИМЕЮЩЕГО СВОИ ЛИНГВИСТИЧЕСКИЕ, КУЛЬТУРНЫЕ, СОЦИАЛЬНЫЕ, ПСИХОЛОГИЧЕСКИЕ И ИНЫЕ СОСТАВЛЯЮЩИЕ, АНАЛИЗ КОТОРЫХ ПОМОЖЕТ УВИДЕТЬ НЕБЕЗЫНТЕРЕСНЫЕ ЗАКОНОМЕРНОСТИ НЕ ТОЛЬКО В СИСТЕМЕ ОТНОШЕНИЙ ВЛАСТИ И ОБЩЕСТВА, НО И ВНУТРИ РАЗЛИЧНЫХ СОЦИАЛЬНЫХ СТРАТ. ТАКОЙ ПОДХОД ДАЕТ ВОЗМОЖНОСТЬ НЕ ПРОСТО ПЕРЕЧИСЛИТЬ «ЭПИТЕТЫ» ВРАГОВ, НО И, ОЧЕВИДНО, ПОЧВУ ДЛЯ РАЗМЫШЛЕНИЙ НАД ПРОБЛЕМОЙ ИДЕНТИФИКАЦИИ И САМОИДЕНТИФИКАЦИИ СОВЕТСКОГО ОБЩЕСТВА.

ИСТОЧНИКОВАЯ БАЗА ИССЛЕДОВАНИЯ ДОСТАТОЧНО СПЕЦИФИЧНА, ЧТО ОБЪЯСНЯЕТСЯ НЕ ТОЛЬКО И НЕ СТОЛЬКО ВИДОВЫМ МНОГООБРАЗИЕМ ИСПОЛЬЗОВАННЫХ ДОКУМЕНТОВ. ПРЕВРАТНОСТИ СОВЕТСКОГО ДЕЛОПРОИЗВОДСТВА И ДОКУМЕНТООБРАЗОВАНИЯ СТАЛИ ПРИЧИНОЙ ФРАГМЕНТАРНОСТИ И НЕСИСТЕМАТИЧНОСТИ (НЕСИСТЕМАТИЗИРОВАННОСТИ) ИСТОЧНИКОВ, РАССРЕДОТОЧЕННЫХ ПО ФОНДАМ РАЗЛИЧНЫХ АРХИВОХРАНИЛИЩ БЫВШЕГО СССР. К ОТСУТСТВИЮ МОНОЛИТНОГО КОМПЛЕКСА ИСТОЧНИКОВ СЛЕДУЕТ ДОБАВИТЬ, ЧТО ДОСТУП КО МНОГИМ ИЗ НИХ ДО СИХ ПОР ЗАТРУДНЕН, И УСИЛИЯ АВТОРА ПО ПРЕОДОЛЕНИЮ ВСЕВОЗМОЖНЫХ ПРЕПЯТСТВИЙ, ВОЗНИКАЮЩИХ НА ПУТИ ИССЛЕДОВАТЕЛЯ В РОССИЙСКИХ АРХИВАХ, ЗАСЛУЖИВАЮТ ВСЯЧЕСКОГО ОДОБРЕНИЯ. НАДОБНО ОТМЕТИТЬ, ЧТО УЖЕ ИЗ ИСТОЧНИКОВОГО ОБЗОРА ЯСНО, ЧТО ФУНДАМЕНТОМ ДЛЯ РАБОТЫ О. СТУППО СТАЛИ МАТЕРИАЛЫ, СОЗДАННЫЕ «ВО ВЛАСТИ» И НАПИСАННЫЕ 
«ВО ВЛАСТЬ». ЗАМЫКАЯСЬ НА ЭТОМ КРУГЕ ИСТОЧНИКОВ, ТАК ИЛИ ИНАЧЕ САНКЦИОНИРОВАННЫХ ВЛАСТЬЮ, АВТОР ЗАГОНЯЕТ СВОЕ ИССЛЕДОВАНИЕ В ОПРЕДЕЛЕННЫЕ РАМКИ - РАМКИ, ОРИЕНТИРОВАННЫЕ НА ДОМИНИРОВАНИЕ ПОДХОДА, В СООТВЕТСТВИИ С КОТОРЫМ СОВЕТСКОЕ ОБЩЕСТВО ОБЪЕКТНО И ВТОРИЧНО ПО ОТНОШЕНИЮ К ВЛАСТИ, А ВЛАСТЬ, СИНОНИМОМ КОТОРОЙ, ПО СУТИ, ЯВЛЯЕТСЯ ЛИБО ЛИЧНО И.В.СТАЛИН, ЛИБО НЕКИЕ ДЕПЕРСОНИФИЦИРОВАННЫЕ «БОЛЬШЕВИКИ», ПО ИСТИНЕ НЕ ЗНАЕТ ГРАНИЦ СВОЕГО ВЛИЯНИЯ. АВТОРСКИЙ ВЫБОР ИСТОЧНИКОВ ГОВОРИТ, СКОРЕЕ, НЕ О ТОМ, ЧТО ЭТИ ИСТОЧНИКИ ФРАГМЕНТАРНЫ, А О ТОМ, ЧТО ИХ ВЫБОР ИСКУССТВЕННО ОГРАНИЧЕН ИЗНАЧАЛЬНО. ЗА «БОРТОМ» РАБОТЫ ПОЧЕМУ-ТО ОСТАЛИСЬ ВПОЛНЕ ДОСТУПНЫЕ ОПУБЛИКОВАННЫЕ МАТЕРИАЛЫ, ФАКТОЛОГИЧЕСКИЙ КАПИТАЛ КОТОРЫХ МОГ БЫ НЕ ТОЛЬКО ЕЕ УКРАСИТЬ, НО И ПОВЫСИТЬ АНАЛИТИЧЕСКИЙ УРОВЕНЬ ${ }^{1}$. ЧАСТУШКИ, АНЕКДОТЫ И ПРОЧЕЕ НЕФОРМАЛЬНОЕ НАРОДНОЕ ТВОРЧЕСТВО ПОМОГЛИ БЫ БОЛЕЕ РЕЛЬЕФНО ОЧЕРТИТЬ ТОТ КРУГ, В КОТОРОМ ВРАЩАЛИСЬ СПУЩЕННЫЕ «СВЕРХУ» ОБРАЗЫ ВРАГА, РАВНО КАК И ПРОЦЕССЫ ИХ МОДИФИКАЦИИ В ОБЩЕСТВЕННОМ СОЗНАНИИ.

СОБСТВЕННО СОДЕРЖАТЕЛЬНАЯ ЧАСТЬ РАБОТЫ СОСТОИТ ИЗ 7 ГЛАВ, КАЖДАЯ ИЗ КОТОРЫХ ДРОБИТСЯ НА НЕСКОЛЬКО ПАРАГРАФОВ (ОТ 2 ДО 7), В РЯДЕ СЛУЧАЕВ ИМЕЮЩИХ ОСОБУЮ ВНУТРЕННЮЮ ГРАДАЦИЮ, ЧТО ПРЕВРАЩАЕТ КНИГУ В СОБРАНИЕ ОЧЕРКОВ, ОБЪЕДИНЕННЫХ ОБЩЕЙ ПРОБЛЕМОЙ. ДВЕ ПЕРВЫЕ ГЛАВЫ КНИГИ НОСЯТ ХАРАКТЕР ПРЕДВАРИТЕЛЬНЫХ, ПРЕТЕНДУЯ НА РОЛЬ ТЕОРЕТИЧЕСКИХ. ОДНАКО ИХ СТАТУС КАК ТАКОВЫХ, ТО ЕСТЬ ТЕОРЕТИЧЕСКИХ, НЕ БЕССПОРЕН. В САМОМ НАЧАЛЕ ПРОБЛЕМА ГЕНЕРАЦИИ ОБРАЗА ВРАГА СВЯЗЫВАЕТСЯ АВТОРОМ С МАРКСИСТСКОЛЕНИНСКОЙ ИДЕЕЙ КЛАССОВОЙ БОРЬБЫ КАК ДВИЖУЩЕЙ СИЛЫ ИСТОРИИ. ТО, ЧТО МАРКСИСТСКО-ЛЕНИНСКОЕ УЧЕНИЕ, АПЕЛЛИРУЯ К НЕИЗБЫВНЫМ СОЦИАЛЬНЫМ ПРОТИВОРЕЧИЯМ, ТРАКТОВАЛО ИСТОРИЮ КАК ВЕЧНЫЙ КОНФЛИКТ ГРУПП АНТАГОНИСТОВ, ИЗВЕСТНО И, БОЛЕЕ ТОГО, ОБЪЯСНИМО ТЕМ ФАКТОМ, ЧТО ЛЮБАЯ ИДЕОЛОГИЯ, ГОСПОДСТВУЮЩАЯ ИЛИ ОППОЗИЦИОННАЯ, НУЖДАЕТСЯ В ПРОТИВНИКЕ. ЭСКАЛАЦИЯ НАСИЛИЯ В ЭПОХУ СТАЛИНИЗМА ИНТЕРЕСНА ТЕМ, ЧТО КАК РЕАЛИЗОВАННАЯ ПЕРСПЕКТИВА ОНА ПРЕДПОЛАГАЕТ КОРРЕЛЯЦИЮ ТЕРМИНАЛЬНЫХ И ИНСТРУМЕНТАЛЬНЫХ ЦЕННОСТЕЙ, ПОЭТОМУ ОБЪЯСНИТЕЛЬНАЯ МОДЕЛЬ ИСТОРИЧЕСКОГО ПРОЦЕССА, СФОРМУЛИРОВАННАЯ К. МАРКСОМ И В.И.ЛЕНИНЫМ, ТРЕБОВАЛА ОБНОВЛЕНИЯ, КОТОРОЕ НАШЛО СВОЕ ВОПЛОЩЕНИЕ В СТАЛИНСКОМ ТЕЗИСЕ «ВРАГ ЖЕСТОК И НЕУМОЛИМ», ОЗНАЧАВШЕМ НЕ ТОЛЬКО ПРОЛОНГИРОВАНИЕ КЛАССОВОЙ БОРЬБЫ В УСЛОВИЯХ ПОБЕДЫ СОЦИАЛИСТИЧЕСКОЙ РЕВОЛЮЦИИ, НО И ЕЕ ОБОСТРЕНИЕ. ПРИХОДЯ К ТАКОМУ ЗАКЛЮЧЕНИЮ, АВТОР ПОДЧЕРКИВАЕТ, ЧТО ГЕНЕРИРОВАННЫЕ В РАННЕМ СТАЛИНИЗМЕ ОБРАЗЫ ВРАГА НЕ УХОДИЛИ В НЕБЫТИЕ, ПОСЛЕДОВАТЕЛЬНО СМЕНЯЯСЬ ОДИН ЗА ДРУГИМ, А ПРЕВРАЩАЛИСЬ В РЕЗЕРВ, ОБРАЗОВЫВАЛИ СВОЕОБРАЗНЫЙ ДЕПОЗИТАРИЙ. «ОТСЛУЖИВШИХ» ОБРАЗОВ ВРАГА НЕ БЫЛО, БЫЛИ УТРАТИВШИЕ АКТУАЛЬНОСТЬ В КОНКРЕТНО-ИСТОРИЧЕСКИХ УСЛОВИЯХ, КОТОРЫЕ, МЕНЯЯСЬ, МОГЛИ ВЫЗЫВАТЬ РЕАНИМАЦИЮ СТАРЫХ ОБРАЗОВ, О ЧЕМ СВИДЕТЕЛЬСТВУЕТ, В ЧАСТНОСТИ, «ДОЛГОЖИТЕЛЬСТВО» ТАКИХ ОБРАЗОВ, КАК «ОСТАТКИ УМИРАЮЩИХ КЛАССОВ» И «РАСТОЧИТЕЛИ СОЦИАЛИСТИЧЕСКОЙ СОБСТВЕННОСТИ».

7 ВО ВТОРОЙ ГЛАВЕ КНИГИ РЕЧЬ ИДЕТ О ТАКОМ ЯВЛЕНИИ, КАК ЩОБЩЕСТВЕННОЕ МНЕНИЕ», А ТОЧНЕЕ О ЕГО СОМНИТЕЛЬНОСТИ В СОВЕТСКИХ УСЛОВИЯХ. СОВЕТСКОЕ ОБЩЕСТВО, ОСНОВАННОЕ НА КОЛЛЕКТИВИСТСКИХ И КОММУНИТАРИСТСКИХ 
ЦЕННОСТЯХ, НЕ ЗНАЛО ИНДИВИДУАЛЬНОЙ АВТОНОМИИ, ГЛАСНОСТИ И ПЛЮРАЛИЗМА, И, СЛЕДОВАТЕЛЬНО, ЦИРКУЛЯЦИЯ ИДЕЙ, НОРМ И СТЕРЕОТИПОВ ОСУЩЕСТВЛЯЛАСЬ ЗДЕСЬ ИНАЧЕ. ОБЩЕСТВЕННОЕ МНЕНИЕ В СССР БЫЛО ПРИНЦИПИАЛЬНО ДРУГИМ, НО ПРИРОДА ДРУГОГО, КАК ПРЕДСТАВЛЯЕТСЯ, СКРЫВАЛАСЬ ПРЕЖДЕ ВСЕГО В ДРУГОМ ХАРАКТЕРЕ ОБЩЕСТВА В ЦЕЛОМ, А, МОЖЕТ БЫТЬ, И В ОТСУТСТВИИ ОБЩЕСТВА ВООБЩЕ В ТОМ ЕГО ПРОЧТЕНИИ, КОТОРОЕ БЫТУЕТ НА ЗАПАДЕ. НО ОТ ОБСУЖДЕНИЯ ЭТОГО ВОПРОСА АВТОР УХОДИТ, ОТСЫЛАЯ ЧИТАТЕЛЯ К ОДНОЙ ИЗ РАБОТ М.ГЕЛЛЕРА 2 . СРАВНЕНИЕ ЖЕ СОЦИАЛЬНЫХ ИНСТИТУТОВ И ПРАКТИК ДЕМОКРАТИЧЕСКОГО МИРА И СТАЛИНСКОГО ОБЩЕСТВА ОБНАРУЖИВАЕТ ИХ МИНИМАЛЬНОЕ СХОДСТВО, А КРИТИЧЕСКОЕ КОЛИЧЕСТВО «ОТКЛОНЕНИЙ» ДОКАЗЫВАЕТ НЕВОЗМОЖНОСТЬ ИХ ТИПИЗАЦИИ, ЧТО СТАЛО ПРИЧИНОЙ ОТКАЗА АВТОРА ОТ ИСПОЛЬЗОВАНИЯ ТЕРМИНА «ОБЩЕСТВЕННОЕ МНЕНИЕ». ВМЕСТО НЕГО ПРЕДЛОЖЕН ТЕРМИН «КОММУНИКАЦИЯ», НО ЕГО ЖЕЛАТЕЛЬНАЯ ПРОБЛЕМАТИЗАЦИЯ, ТАК ЖЕ КАК И ТЕМАТИЗАЦИЯ ПОНЯТИЯ «ОБРАЗ ВРАГА» ${ }^{3}$, В РАБОТЕ ОПУЩЕНА.

ГЬЯ ГЛВА КНИГИ ПОСВЯЩЕНА ХАРАКТЕРИСТИКЕ СВЕРДЛОВСКОЙ ОБЛАСТИ, ОТДАЛЕННОСТЬ КОТОРОЙ ОТ ЕВРОПЕЙСКОЙ ЧАСТИ СССР ОДНОВРЕМЕННО БЫЛА И ПРЕИМУЩЕСТВОМ, И НЕДОСТАТКОМ РЕГИОНА. КАК ЦЕНТР ПРОИЗВОДСТВА СОВЕТСКОГО АТОМНОГО ОРУЖИЯ ОБЛАСТЬ ПРЕДСТАВЛЯЛА СОБОЙ ИЗОЛИРОВАННУЮ ЗОНУ, ВЪЕЗД И ВЫЕЗД ИЗ КОТОРОЙ БЫЛ ОГРАНИЧЕН. СТРОГО ГОВОРЯ, ОДНАКО, ГОВОРИТЬ О ГОРОДЕ СВЕРДЛОВСКЕ КАК О ГОРОДЕ ЗАКРЫТОМ БЫЛО БЫ НЕ СОВСЕМ ВЕРНО. ФОРМАЛЬНО ОН ТАКОВЫМ НЕ ЯВЛЯЛСЯ, КАК И ПОДАВЛЯЮЩЕЕ БОЛЬШИНСТВО ГОРОДОВ ОБЛАСТИ, ЗА ИСКЛЮЧЕНИЕМ НЫНЕШНИХ НОВОУРАЛЬСКА И ЛЕСНОГО, СОХРАНИВШИХ ЗА СОБОЙ СТАТУС ЗАКРЫТЫХ ДО СЕГОДНЯШНЕГО ДНЯ ${ }^{4}$. ТРЕБУЕТ СТАТИСТИЧЕСКОГО ПОДТВЕРЖДЕНИЯ И ТЕЗИС АВТОРА О КОНЦЕНТРАЦИИ В СВЕРДЛОВСКОЙ ОБЛАСТИ БОЛЬШОГО ЧИСЛА ЗАКЛЮЧЕННЫХ, В ПРИНУДИТЕЛЬНОМ ПОРЯДКЕ ВОВЛЕКАЕМЫХ В ЭКОНОМИЧЕСКИЕ ПРОЦЕССЫ, ЧТО СОСТАВЛЯЛО ВТОРУЮ ОСОБЕННОСТЬ РАССМАТРИВАЕМОГО РЕГИОНА.

ВСЛЕД ЗА АБРИСОМ СПЕЦИФИКИ СВЕРДЛОВСКОЙ ОБЛАСТИ О.СТУППО ВОССОЗДАЕТ ИСТОРИЮ УРАЛЬСКОГО РАБОЧЕГО КЛАССА, ЗАСТАВЛЯЯ ЧИТАТЕЛЯ ВСПОМНИТЬ ОСНОВНЫЕ ВЕХИ РОССИЙСКОЙ ИСТОРИИ - ПЕРВУЮ РУССКУЮ РЕВОЛЮЦИЮ И РЕВОЛЮЦИЮ 1917 ГОДА, ГРАЖДАНСКУЮ ВОЙНУ И ПРОТИВОРЕЧИВЫЙ НЭП, СТАВШИЙ ПРЕДДВЕРИЕМ ИНДУСТРИАЛИЗАЦИИ, И, СОБСТВЕННО, САМУ ИНДУСТРИАЛИЗАЦИЮ, В ХОДЕ КОТОРЫХ В СОЗНАНИЕ РАБОЧИХ ПОСЛЕДОВАТЕЛЬНО ВЖИВЛЯЛИСЬ ОБРАЗЫ «ЭКС-БЕЛОГВАРДЕЙЦА», «ТРОЦКИСТА», «ЛИШЕНЦА», «БУРЖУАЗНОГО ИНЖЕНЕРА», «РУКОВОДИТЕЛЯ ВРЕДИТЕЛЯ» И ДР. ПРИ ЭТОМ ОЧЕВИДНО, ЧТО РОЖДЕНИЕ ТОГО ИЛИ ИНОГО ОБРАЗА ВРАГА БЫЛО СВЯЗАНО С ТЕМИ ИЛИ ИНЫМИ АКТУАЛЬНЫМИ ЗАДАЧАМИ РАЗВИТИЯ СТРАНЫ, РЕШАТЬ КОТОРЫЕ ЭФФЕКТИВНО ВЛАСТЬ БЫЛА НЕСПОСОБНА, СПИСЫВАЯ ВСЕ НЕУДАЧИ НА ПРОИСКИ ВРАГА, ПОИСК КОТОРЫХ СТАЛ В ИТОГЕ ВАЖНЕЙШИМ ЗАНЯТИЕМ НЕ ТОЛЬКО ПОЛИТИЧЕСКОЙ ЭЛИТЫ, НО И НАСЕЛЕНИЯ. НЕЗРЕЛОСТЬ РАБОЧЕГО КЛАССА, ЕГО ПОЛИТИЧЕСКАЯ ПАССИВНОСТЬ БЫЛИ КАТАЛИЗАТОРОМ ПРОЦЕССА УСВОЕНИЯ ОБРАЗОВ ВРАГА В ГОРОДЕ, ВЫТЕСНЕНИЯ ИЗ СОЗНАНИЯ РАБОЧИХ РАЦИОНАЛЬНЫХ ПРИЕМОВ ОБЪЯСНЕНИЯ ПРОТИВОРЕЧИЙ. ОТКАЗ ОТ ВЫЯВЛЕНИЯ РЕАЛЬНЫХ ПРИЧИН НЕСООТВЕТСТВИЯ МЕЖДУ ПРОВОЗГЛАШЕННЫМИ СОЦИАЛЬНО-ЭКОНОМИЧЕСКИМИ ИДЕАЛАМИ И ИХ ОБЪЕКТИВИРОВАННЫМИ ПРОЯВЛЕНИЯМИ НИКАК НЕ БЛАГОПРИЯТСТВОВАЛ ПРЕОДОЛЕНИЮ НАСУЩНЫХ СОЦИАЛЬНО-ЭКОНОМИЧЕСКИХ ПРОБЛЕМ, СКОРЕЕ, НАОБОРОТ. И ПУГАЮЩАЯ 
МНОЖЕСТВЕННОСТЬ ЛИКОВ ВРАГА, ПО-ВИДИМОМУ, ОТРАЖАЛА ПРОЦЕСС НАСЛОЕНИЯ ВНОВЬ ВОЗНИКАЮЩИХ ПРОТИВОРЕЧИЙ НА УЖЕ СУЩЕСТВУЮЩИЕ НЕРАЗРЕШЕННЫЕ ПРОТИВОРЕЧИЯ, ПРЕВРАЩАЯ СОЦИАЛЬНОЕ ПРОСТРАНСТВО В СВОЕГО РОДА СЛОЖНО СТРУКТУРИРОВАННОЕ КОНФЛИКТНОЕ ПОЛЕ.

10 НА СЕЛЕ, ГДЕ В ГОДЫ КОЛЛЕКТИВИЗАЦИИ ПОЛУЧИЛ РАСПРОСТРАНЕНИЕ ПАРАДОКСАЛЬНО ЖИВУЧИЙ ОБРАЗ «КУЛАКА», КОТОРЫЙ, КАЗАЛОСЬ БЫ, БЫЛ ОБРЕЧЕН НА ОТМИРАНИЕ С ЛИКВИДАЦИЕЙ КУЛАКА КАК КЛАССА, В ПОСТВОЕННЫЙ ПЕРИОД АКТИВНО НАСАЖДАЛСЯ ОБРАЗ «ВРАГА КОЛХОЗНОГО СТОЯ», ПРЕДСТАВЛЯВШИЙ СОБОЙ КОМБИНАЦИЮ ЭЛЕМЕНТОВ ОБРАЗА «КУЛАКА» И ДРУГИХ СКОНСТРУИРОВАННЫХ РАНЕЕ «ПОДОБРАЗОВ». АНАЛИЗ АДРЕСОВАННЫХ «ВО ВЛАСТЬ» ЖАЛОБ И ДОНОСОВ КОЛХОЗНИКОВ, ПРОВЕДЕННЫЙ АВТОРОМ, СВИДЕТЕЛЬСТВУЕТ, ЧТО НА ФОНЕ БЕЗОШИБОЧНО ВОСПРОИЗВЕДЕННЫХ КЛИШИРОВАННЫХ ХАРАКТЕРИСТИК ВРАГА ОСТАЛЬНОЙ ТЕКСТ ИЗОБИЛОВАЛ ГРАММАТИЧЕСКИМИ ОШИБКАМИ. ПОЭТОМУ ЕДВА ЛИ БУДЕТ ПРЕУВЕЛИЧЕНИЕМ СКАЗАТЬ, ЧТО В ДЕРЕВНЕ РЕЛИКТОВАЯ ПО СВОЕЙ ПРИРОДЕ ПРАКТИКА ПОИСКА ВИНОВАТОГО ВО ВСЕХ ВСЕЛЕНСКИХ БЕДАХ «КОЗЛА ОТПУЩЕНИЯ» РАБОТАЛА ЭФФЕКТИВНЕЕ, ЧЕМ В ГОРОДЕ, В СИЛУ СОХРАНЕНИЯ АРХАИЧЕСКИХ ЦЕННОСТЕЙ СЕЛЬСКОЙ ОБЩИНЫ, ОРИЕНТИРОВАННОЙ НА НАТУРАЛЬНОЭКОНОМИЧЕСКИЙ» ПОРЯДОК. АВТОР ПОДЧЕРКИВАЕТ, ЧТО КОЛЛЕКТИВИЗАЦИЯ, СТАЛКИВАЯ МЕЖДУ СОБОЙ РАЗНЫЕ УКЛАДЫ КРЕСТЬЯНСКОЙ ЖИЗНИ, НЕ ПРИВЕЛА К ИЗЖИВАНИЮ ТРАДИЦИОННЫХ ПРЕДСТАВЛЕНИЙ О «ВСЕОБЩЕМ БЛАГЕ» И «МОРАЛЬНОЙ ЭКОНОМИКЕ», КОТОРЫЕ ОБРАТИЛИСЬ, В КОНЕЧНОМ СЧЕТЕ, ПРОТИВ ПРЕДСТАВИТЕЛЕЙ САМОЙ ВЛАСТИ, В ЧАСТНОСТИ, «ЗАСЛАННЫХ» ИЗВНЕ КОЛХОЗНОГО МИРА ПРЕДСЕДАТЕЛЕЙ КОЛХОЗОВ, ОСТАВАВШИХСЯ ДЛЯ КОЛХОЗНИКОВ ЧУЖИМИ И УЖЕ ПОТОМУ ВРАЖДЕБНЫМИ. «БЕСФОРМЕННОСТЬ» ОБРАЗА ВРАГА В ЭТОМ, КАК И В ДРУГИХ СЛУЧАЯХ, ПОЗВОЛЯЛА ВПИСАТЬ В ЕГО РАМКИ ДАЖЕ ТЕХ, КТО УЧАСТВОВАЛ В ЕГО СОЗДАНИИ И РЕЗОНИРОВАНИИ, ДОСТАТОЧНО БЫЛО ЛИШЬ ОДНАЖДЫ ВЫКАЗАТЬ ТАКИЕ СЛАБОСТИ, КАК «ХИТРОСТЬ» И «КОРЫСТОЛЮБИЕ», ИМЕТЬ «СОЦИАЛЬНО-ЧУЖДОЕ» ПРОИСХОЖДЕНИЕ ИЛИ НАМЕРЕННО ИЛИ НЕТ «ПЕРЕЙТИ ДОРОГУ» СОСЕДУ, СТАВ ОБЪЕКТОМ ЕГО МЕСТИ.

ПРОБЛЕМА ЛИЧНЫХ АНТИПАТИЙ КАК ФАКТОРА, ВЛИЯВШЕГО НА КОММУНИКАТИВНЫЙ ОПЫТ, ЗАТРАГИВАЕТСЯ В ГЛАВЕ, ПОСВЯЩЕННОЙ МЕСТУ И РОЛИ ИНТЕЛЛИГЕНЦИИ В БОРЬБЕ ЗА «СВЕТЛЫЕ» КОММУНИСТИЧЕСКИЕ ИДЕАЛЫ. СОВЕТСКАЯ ИНТЕЛЛИГЕНЦИЯ, КОНСТАТИРУЕТ АВТОР ВСЛЕД ЗА В.И. ЛЕНИНЫМ, НЕ МОГЛА ПРЕТЕНДОВАТЬ НИ НА КАКУЮ САМОСТОЯТЕЛЬНУЮ ПОЛИТИЧЕСКУЮ ВОЛЮ И СИЛУ, ЯВЛЯЯСЬ НЕ БОЛЕЕ ЧЕМ «ПРОСЛОЙКОЙ», ПРИЗВАННОЙ ЦЕМЕНТИРОВАТЬ СОЮЗ ПРОЛЕТАРИАТА И КРЕСТЬЯНСТВА, ОФОРМЛЯЯ И ТРАНСЛИРУЯ ИДЕОЛОГИЧЕСКИЕ ИМПЕРАТИВЫ, В ТОМ ЧИСЛЕ И ОБРАЗЫ ВРАГА. ОДНАКО ТАКОЙ ПОДХОД ФАКТИЧЕСКИ ПРЕДПОЛАГАЛ СЕРЬЕЗНУЮ ТРАНСФОРМАЦИЮ, ЕСЛИ НЕ СОЗДАНИЕ НОВОЙ ИНТЕЛЛЕКТУАЛЬНОЙ ЭЛИТЫ СОВЕТСКОГО ОБЩЕСТВА. НА ПРИМЕРЕ УРАЛЬСКОГО ГОСУДАРСТВЕННОГО УНИВЕРСИТЕТА, УРАЛЬСКОГО ФИЛИАЛА АН СССР, СВЕРДЛОВСКОГО ЮРИДИЧЕСКОГО ИНСТИТУТА, СВЕРДЛОВСКОГО ТЕАТРАЛЬНОГО ИНСТИТУТА И СВЕРДЛОВСКОЙ ГОСУДАРСТВЕННОЙ МУЗЫКАЛЬНОЙ КОНСЕРВАТОРИИ АВТОР ПОКАЗЫВАЕТ, КАКОВЫ БЫЛИ ОСНОВНЫЕ МЕТОДЫ БОРЬБЫ ЗА ИДЕЙНОСТЬ СОВЕТСКОГО ОБРАЗОВАНИЯ, НАУКИ И КУЛЬТУРЫ. ИСПОЛЬЗУЯ ЛАТЕНТНЫЙ АНТИСЕМИТИЗМ И РУССКИЙ НАЦИОНАЛИЗМ, ВЛАСТЬ СФОРМИРОВАЛА И ВНЕДРИЛА В СОЗНАНИЕ ЛЮДЕЙ ОТРИЦАТЕЛЬНЫЙ ОБРАЗ «БЕЗРОДНОГО» КОСМОПОЛИТА, СВОДЯ ТЕМ САМЫМ ЛЮБЫЕ СВОБОДНЫЕ ПРОЯВЛЕНИЯ 
ТВОРЧЕСКОЙ МЫСЛИ К КОНТРОЛИРУЕМОМУ МИНИМУМУ. ТАКОГО РОДА КОНФИГУРАЦИЯ ОТНОШЕНИЙ В СРЕДЕ, ГДЕ ДИСКУССИЯ ВСЕГДА БЫЛА ВАЖНЕЙШИМ ЭЛЕМЕНТОМ КОММУНИКАЦИИ, ЗАСТАВЛЯЛА ЛЮДЕЙ ТВОРЧЕСКИХ СПЕЦИАЛЬНОСТЕЙ ВМЕСТО ВЫПОЛНЕНИЯ ИХ НЕПОСРЕДСТВЕННЫХ ПРОФЕССИОНАЛЬНЫХ ОБЯЗАННОСТЕЙ ПОСТОЯННО ДОКАЗЫВАТЬ СВОЮ ЛОЯЛЬНОСТЬ ВЛАСТИ И ГОСПОДСТВУЮЩЕМУ ПОЛИТИЧЕСКОМУ (И НЕ ТОЛЬКО ПОЛИТИЧЕСКОМУ) ДИСКУРСУ, ДОНОСЯ НА КОЛЛЕГ И ОБЛИЧАЯ ИХ НА СОБРАНИЯХ. ИНТЕРВЕНЦИЯ ГОСУДАРСТВА В СФЕРУ ТВОРЧЕСТВА ПРЕВРАЩАЛА ТВОРЧЕСТВО В ИМИТАЦИЮ, НАУКУ - В «ИДЕОЛОГИЧЕСКИЙ МАРАЗМ», А ИДЕЮ СОЮЗА НАУКИ И ПРОИЗВОДСТВА - В ХИМЕРУ. ОТКАЗЫВАЯ ИНТЕЛЛИГЕНЦИИ В РОЛИ КОММУНИКАТИВНОГО ПОСРЕДНИЧЕСТВА МЕЖДУ ГРУППАМИ, ТОЧНЕЕ, ПОДМЕНЯЯ ЕЕ СЕРВИЛЬНОЙ ФУНКЦИЕЙ ПОСРЕДНИЧЕСТВА ИДЕОЛОГИЧЕСКОГО, ВЛАСТЬ НЕ ПРОСТО ЛИШАЛА ЕЕ МАНЕВРА, НО И ПРЕВРАЩАЛА В СВОЮ ЖЕРТВУ, СТАВЯ ЕЕ КАК СОЦИАЛЬНУЮ ЕДИНИЦУ НА ГРАНЬ ВЫЖИВАНИЯ. ОСТАЕТСЯ ТОЛЬКО СОЖАЛЕТЬ, ЧТО АВТОР НИКАК НЕ КОММЕНТИРУЕТ ДАВНО ИДУЩУЮ ДИСКУССИЮ О ТОМ, ЧТО ТАКОЕ СОВЕТСКАЯ ИНТЕЛЛИГЕНЦИЯ И ИНТЕЛЛИГЕНЦИЯ ЛИ ЭТО ВООБЩЕ?

СЮЖЕТНАЯ ЛИНИЯ ШЕСТОЙ ГЛАВЫ КНИГИ ПОСТРОЕНА НА РАССМОТРЕНИИ ПРОБЛЕМЫ ВОСПРИЯТИЯ СОВЕТСКИМ ОБЩЕСТВОМ ИСКУССТВЕННО ИНТЕГРИРОВАННОЙ В ЕГО СОСТАВ ПОСЛЕ ВОЙНЫ ГРУППЫ РЕПАТРИАНТОВ ИЗ КИТАЯ, ПО ПРЕИМУЩЕСТВУ РУССКИХ. ИХ ДОБРОВОЛЬНО-ПРИНУДИТЕЛЬНОЕ ВОЗВРАЩЕНИЕ НА ИСТОРИЧЕСКУЮ РОДИНУ, ПО МНЕНИЮ АВТОРА, БЫЛО СВЯЗАНО С РЕШИМОСТЬЮ РЕЖИМА ПОКОНЧИТЬ С БЕЛОЙ ЭМИГРАЦИЕЙ И ВОСПРЕПЯТСТВОВАТЬ ПРОНИКНОВЕНИЮ В СТРАНУ АМЕРИКАНСКОГО И ЯПОНСКОГО ВЛИЯНИЯ, КОТОРОЕ МОГЛО КАНАЛИЗИРОВАТЬСЯ ЧЕРЕЗ ТАК НАЗЫВАЕМУЮ РУССКУЮ МАНЬЧЖУРИЮ. НО МОТИВЫ СОВЕТСКОГО РУКОВОДСТВА ЕДВА ЛИ СОСТОЯЛИ ЛИШЬ В ЭТОМ. НЕ СЕКРЕТ, ЧТО ПОСЛЕ ВОЙНЫ АВТОРИТЕТ СССР НА МЕЖДУНАРОДНОЙ АРЕНЕ БЫЛ ДОСТАТОЧНО ВЫСОК, И МНОГИЕ СООТЕЧЕСТВЕННИКИ ЗА РУБЕЖОМ ИСКРЕННЕ ВЕРИЛИ В СВОЮ РОДИНУ И СТРЕМИЛИСЬ ОБРЕСТИ ЕЕ СНОВА, ЧЕМ СТАЛИНСКОЕ ПРАВИТЕЛЬСТВО НЕ МОГЛО НЕ ВОСПОЛЬЗОВАТЬСЯ, ПОДАВАЯ МАССОВОЕ ВОЗВРАЩЕНИЕ БЫВШИХ РОССИЯН ДОМОЙ (В ТОМ ЧИСЛЕ ИЗ СТРАН ЕВРОПЫ) КАК АРГУМЕНТ В ПОЛЬЗУ ПРЕВОСХОДСТВА СОЦИАЛИСТИЧЕСКОГО СТРОЯ НАД КАПИТАЛИСТИЧЕСКИМ. ЭФФЕКТ ВНЕШНИЙ В ДАННОМ СЛУЧАЕ БЫЛ ГОРАЗДО ВАЖНЕЕ, ЧЕМ ПРАГМАТИЧЕСКИЕ СООБРАЖЕНИЯ ЧАСТНОГО СВОЙСТВА, К ЧИСЛУ КОТОРЫХ АВТОР ОТНОСИТ ЗАИНТЕРЕСОВАННОСТЬ СОВЕТСКОЙ ЭКОНОМИКИ В ДОПОЛНИТЕЛЬНЫХ ВЛИВАНИЯХ РАБОЧИХ РУК. С ТОЧКИ ЗРЕНИЯ ВНУТРИПОЛИТИЧЕСКОЙ, РЕПАТРИАНТЫ, НА САМОМ ДЕЛЕ, ОКАЗЫВАЛИСЬ СЛИШКОМ ХЛОПОТНЫМ «ХОЗЯЙСТВОМ», НО ГЛАВНАЯ ЦЕЛЬ, ЧТО НАЗЫВАЕТСЯ, ОПРАВДЫВАЛА ЛЮБЫЕ ИЗДЕРЖКИ. ВМЕСТЕ С ТЕМ, АВТОР, КОНЕЧНО ЖЕ, ПРАВ, УКАЗЫВАЯ НА ТО, ЧТО РЕПАТРИАНТЫ КАК «НЕ ЗАСЛУЖИВАЮЩИЕ ДОВЕРИЯ ЛИЦА» БЫЛИ ПРИНЯТЫ НА РОДИНЕ ВЕСЬМА ХОЛОДНО, НА УРОВНЕ ОБЫВАТЕЛЯ ОСТАВАЯСЬ ИНОСТРАНЦАМИ, АНТИСОВЕТСКОЕ ПРОШЛОЕ КОТОРЫХ СТАЛО ПРИЧИНОЙ ИХ ОТТОРЖЕНИЯ НАСЕЛЕНИЕМ И УГОЛОВНОГО ПРЕСЛЕДОВАНИЯ СО СТОРОНЫ «ЗЛОПАМЯТНЫХ» ВЛАСТЕЙ.

ФИНАЛЬНАЯ ЧАСТЬ ИССЛЕДОВАНИЯ СФОКУСИРОВАНА НА ПРОБЛЕМЕ ПРОДУЦИРОВАНИИ ОБРАЗА ЭТНИЧЕСКОГО ВРАГА И ЕГО ЦИРКУЛЯЦИИ В МАССОВОМ СОЗНАНИИ. ПРИМЕРОМ ДЛЯ РАССМОТРЕНИЯ ЭТОГО АСПЕКТА ТЕМЫ ВЫБРАНЫ НЕМЦЫ. НО, ОГРАНИЧИВАЯСЬ АНАЛИЗОМ СОБЫТИЙ ПОСЛЕВОЕННОГО ВРЕМЕНИ, АВТОР ЯВНО УПУСКАЕТ ИЗ ВИДУ ТОТ ФАКТ, ЧТО НЕГАТИВНЫЙ ОБРАЗ РОССИЙСКОГО НЕМЦА, НЕ ГОВОРЯ УЖЕ О НЕМЕЦКИХ ВОЕННОПЛЕННЫХ, СЛОЖИЛСЯ В ОСНОВНЫХ СВОИХ ЧЕРТАХ КАК МИНИМУМ ДО 1945 Г. ${ }^{5}$ 
НЕДОУМЕНИЕ ВЫЗЫВАЕТ И ТО, ЧТО МЕЖДУ ТРУДМОБИЛИЗОВАННЫМИ НЕМЦАМИ И НЕМЕЦКИМИ ВОЕННОПЛЕННЫМИ, ЧЬЕ ПОЛОЖЕНИЕ, КАК И ВОСПРИЯТИЕ В ОБЩЕСТВЕ, БЫЛО ПРИНЦИПИАЛЬНО ИНЫМ, ПОСКОЛЬКУ ПЕРВЫЕ ВСЕ-ТАКИ БЫЛИ НЕМЦАМИ «СВОИМИ», А ВТОРЫЕ - ЗАХВАТЧИКАМИ, АВТОР НЕ ПРОВОДИТ ЧЕТКОЙ ГРАНИ. ДОСАДНО, НО В ЭТОЙ СВЯЗИ АВТОРСКАЯ ПОПЫТКА «СОСЧИТАТЬ» НЕМЦЕВ, ПРЕБЫВАВШИХ В ПОСЛЕВОЕННЫЙ ПЕРИОД НА ТЕРРИТОРИИ СВЕРДЛОВСКОЙ ОБЛАСТИ, ВЫГЛЯДИТ ВЕСЬМА СОМНИТЕЛЬНО. СОМНИТЕЛЕН ТАКЖЕ И ТЕЗИС О НЕЭФФЕКТИВНОСТИ ТРУДОИСПОЛЬЗОВАНИЯ НЕМЦЕВ В ЭКОНОМИКЕ РЕГИОНА; ОН, С НЕКОТОРЫМИ ОГОВОРКАМИ, ДОПУСТИМ В ОТНОШЕНИИ ВОЕННОПЛЕННЫХ, В ТО ВРЕМЯ КАК РОССИЙСКИЕ НЕМЦЫ ТРУДИЛИСЬ САМООТВЕРЖЕННО, НЕСМОТРЯ НА ТУ НЕСПРАВЕДЛИВОСТЬ, КОТОРОЙ ОНИ БЫЛИ «УДОСТОЕНЫ» СТАЛИНСКИМ РЕЖИМОМ. ПАССАЖ О ВРАЖДЕБНОМ ОТНОШЕНИИ МЕСТНЫХ ЖИТЕЛЕЙ К НЕМЦАМ-ТРУДАРМЕЙЦАМ (ПОСЛЕ ВОЙНЫ - СПЕЦПОСЕЛЕНЦАМ) И ДРУЖЕЛЮБНОМ ОТНОШЕНИИ К ВОЕННОПЛЕННЫМ ТАКЖЕ УМОЗРИТЕЛЕН И ТРЕБУЕТ ДОПОЛНИТЕЛЬНОЙ АРГУМЕНТАЦИИ. БЛИЖЕ К ПРАВДЕ ВСЕ-ТАКИ БУДЕТ ТО, ЧТО КАК «ПЛОХОЙ НАРОД» «АБОРИГЕНАМИ» ВОСПРИНИМАЛИСЬ НЕМЦЫ, ПРИВЕЗЕННЫЕ НА УРАЛ В ВОЙНУ, С ЗАВЕРШЕНИЕМ КОТОРОЙ, ОДНАКО, АНТИНЕМЕЦКИЕ НАСТРОЕНИЯ ПОШЛИ НА СПАД. ЗАКРЫВАЯ КНИГУ, ХОЧЕТСЯ СОГЛАСИТЬСЯ С ВЫВОДОМ АВТОРА О ТОМ, ЧТО, ИСПОЛЬЗУЯ ОБРАЗ ВРАГА КАК СРЕДСТВО ДИСЦИПЛИНИРОВАНИЯ, МОБИЛИЗАЦИИ И КОНСОЛИДАЦИИ НАСЕЛЕНИЯ ПЕРЕД ЛИЦОМ РЕАЛЬНОЙ ИЛИ ГИПОТЕТИЧЕСКОЙ УГРОЗЫ, СТАЛИНСКИЙ РЕЖИМ, СКОРЕЕ, СПОСОБСТВОВАЛ ДЕЗИНТЕГРАЦИИ ОБЩЕСТВА И ДЕГРАДАЦИИ ОБЩЕСТВЕННЫХ ИНСТИТУТОВ. ПАРАДОКСАЛЬНОСТЬ СТАЛИНСКОЙ СОЦИАЛЬНОЙ ПОЛИТИКИ ДОБИВАТЬСЯ СПЛОЧЕНИЯ ПОСРЕДСТВОМ СЕГРЕГАЦИИ НЕОСПОРИМА, КАК НЕОСПОРИМ КУМУЛЯТИВНЫЙ ХАРАКТЕР АКТИВНО ПРОИЗВОДИМЫХ И ЭКСПЛУАТИРУЕМЫХ В ТО ВРЕМЯ ОБРАЗОВ ВРАГА. ДУМАЕТСЯ, ОДНАКО, ЧТО АМОРФНОСТЬ И РАСТЯЖИМОСТЬ (В ТОМ ЧИСЛЕ ВРЕМЕННАЯ) ТЕХ ИЛИ ИНЫХ СОЦИАЛЬНО КОНСТРУИРУЕМЫХ ОБРАЗОВ ВРАГА ЕСТЬ НИЧТО ИНОЕ, КАК ПОКАЗАТЕЛЬ ИХ УНИВЕРСАЛЬНОЙ ФОРМЫ, А НЕ ИХ ПРОБЛЕМАТИЧНОЙ ИСЧИСЛЯЕМОСТИ. ОБРАЗ ВРАГА КАК СИМВОЛ, ФАНТОМ ИЛИ КОД, ЛИШЕННЫЙ СВОЕЙ ПРЕДМЕТНОЙ КОНКРЕТНОСТИ, НАПОЛНЯЛСЯ СОДЕРЖАНИЕМ, ЧТО НАЗЫВАЕТСЯ, ПО СИТУАЦИИ, В ТО ВРЕМЯ КАК ЕГО КОНТУРЫ В ЦЕЛОМ ОСТАВАЛИСЬ ТЕМИ ЖЕ. ОТСЮДА НЕВОЗМОЖНОСТЬ ЧЕТКОГО РАЗГРАНИЧЕНИЯ ОБРАЗОВ, ТКАНЬ КОТОРЫХ БЫЛА ОДНОЙ И ТОЙ ЖЕ, А РИСУНОК МЕНЯЛСЯ В ЗАВИСИМОСТИ ОТ СМЕНЫ ЕЖЕМОМЕНТНЫХ ВНЕШНЕ- И ВНУТРИПОЛИТИЧЕСКИХ, ЭКОНОМИЧЕСКИХ И СОЦИАЛЬНЫХ ЗАДАЧ. КСТАТИ, ИМЕННО РАСПЛЫВЧАТОСТЬ ОБРАЗА ВРАГА ПРЕВРАЩАЛА ЕГО В ДЕЙСТВЕННОЕ СРЕДСТВО МАНИПУЛЯЦИИ ОБЩЕСТВЕННЫМ СОЗНАНИЕМ. НА КОММУНИКАТИВНОМ УРОВНЕ ЭТО, В ЧАСТНОСТИ, ПРОЯВЛЯЛОСЬ В ТОМ, ЧТО ОБРАЗ, ЗАДАВЛЕННЫЙ И СТЕСНЕННЫЙ МНОЖЕСТВОМ СМЫСЛОВ, МОГ ИХ ВООБЩЕ ЛИШАТЬСЯ И НАЧИНАЛ ИХ САМОСТОЯТЕЛЬНО ПОРОЖДАТЬ И РЕДУЦИРОВАТЬ, ПРИ УМЕЛОМ С НИМ ОБРАЩЕНИИ БРОСАЯ ТЕНЬ ВРАГА ПРАКТИЧЕСКИ НА ЛЮБОГО. ПОЭТОМУ МЕЖДУ ОБРАЗОМ ВРАГА В ПРОПАГАНДЕ И В ИНДИВИДУАЛЬНОМ СОЗНАНИИ НЕРЕДКО ПРОЛЕГАЛА ЗНАЧИТЕЛЬНАЯ ДИСТАНЦИЯ, ЧТО НЕ МЕШАЛО ЕМУ БЫТЬ СВОЕГО РОДА МОСТИКОМ МЕЖДУ ПРАВИТЕЛЯМИ И УПРАВЛЯЕМЫМИ, НО В ТО ЖЕ ВРЕМЯ ВЫТЕСНЯЛО НА ПЕРИФЕРИЮ ДРУГИЕ СРЕДСТВА КОММУНИКАЦИИ, НИКАК, К СОЖАЛЕНИЮ, НЕ АРТИКУЛИРОВАННЫЕ АВТОРОМ. 
НАКОНЕЦ, СТОИТ ЗАКЛЮЧИТЬ, ЧТО РАБОТА О.СТУППО ПРИ ЕЕ ФУНДИРОВАННОСТИ И, СКОРЕЕ, ПАНОРАМНОМ, НЕЖЕЛИ РЕГИОНАЛЬНОМ ВИДЕНИИ ЗАВЯЛЕННОЙ ПРОБЛЕМАТИКИ НЕ ТОЛЬКО СТАНЕТ ПОВОДОМ ДЛЯ РАЗДУМИЙ НЕБОЛЬШОГО КРУГА «ПОСВЯЩЕННЫХ» СПЕЦИАЛИСТОВ, НО И БУДЕТ ИНТЕРЕСНА ВСЕМ, КТО ЗАНИМАЕТСЯ ИСТОРИЕЙ, ЭКОНОМИКОЙ, СОЦИОЛОГИЕЙ И ПСИХОЛОГИЕЙ СОВЕТСКОГО ОБЩЕСТВА.

\section{NOTES}

1. СМ., НАПР. А.А. КАПУСТИН И Г.Е. КОРНИЛОВ, ОТВ. РЕД., ОБЩЕСТВО И ВЛАСТЬ. РОССИЙСКАЯ ПРОВИНЦИЯ. 1917-1941. СВЕРДЛОВСКАЯ ОБЛАСТЬ. ДОКУМЕНТЫ И МАТЕРИАЛЫВ 2-Х ТТ., ЕКАТЕРИНБУРГ, 2005, 2006; КОЛХОЗНАЯ ЖИЗНЬ НА УРАЛЕ. 1935-1953 СОСТ. Х. КЕССЛЕР, Г.Е. КОРНИЛОВ. СЕР.: ДОКУМЕНТЫ СОВЕТСКОЙ ИСТОРИИ. М., 2006; НЕМЦЫ В ПРИКАМЬЕ. ХХ ВЕК: СБ. ДОКУМЕНТОВВ 2-Х ТТ., ПЕРМЬ, 2006.

2. М. ГЕЛЛЕР, МАШИНА И ВИНТИКИ: ИСТОРИЯ ФОРМИРОВАНИЯ СОВЕТСКОГО ЧЕЛОВЕКА, М., 1994.

3. РАСШИФРОВКА ТЕРМИНА «ОБРАЗ ВРАГА» ПОЯВЛЯЕТСЯ ТОЛЬКО ВО ВТОРОЙ ПОЛОВИНЕ ТЕКСТА, В ПЯТОЙ ГЛАВЕ (С. 139 КНИГИ), ПРИЧЕМ В ВИДЕ цИТАТЫ ИЗ РАБОТЫ: S.S. Satjukow, R. Gries (hgg.), Unsere Feinde. Konstruktionen des Anderen im Sozialismus,Leipzig, 2004.

4. СМ. ОБ ЭТОМ: Н.В. МЕЛЬНИКОВА, ФЕНОМЕН ЗАКРЫТОГО АТОМНОГО ГОРОДА СЕР.: ОЧЕРКИ ИСТОРИИ УРАЛА, ВЫП. 42, ЕКАТЕРИНБУРГ, 2006.

5. ЕСТЬ ОСНОВАНИЯ ПОЛАГАТЬ, ЧТО НЕГАТИВНЫЙ ОБРАЗ НЕМЦА НЕ СТАЛ ИЗОБРЕТЕНИЕМ СТАЛИНСКОГО РЕЖИМА. СМ. ОБ ЭТОМ, НАПР.: Е.С. СЕНЯВСКАЯ, ОБРАЗ ВРАГА В СОЗНАНИИ УЧАСТНИКОВ ПЕРВОЙ МИРОВОЙ ВОЙНЫМ», ВОПРОСЫ ИСТОРИИ, 3, 1997, С. 140-145. 